\title{
Colección de Bioética. Una nueva contribución en Archivos
}

Bioethics colection. A new contribution in Archivos

Subcomisión de Ética Clínica*

http:/ /dx.doi.org/10.5546/aap.2015.473

Archivos Argentinos de Pediatría es la publicación pediátrica más importante y prestigiosa de nuestro país y un orgullo para la comunidad de pediatras y aliados de la salud infantil. Además de en Scielo® es una de las nueve publicaciones argentinas indexadas en Pubmed $®$.

De ellas, Archivos es la de más prolongada tradición, ya que se publica sin interrupción desde 1930, seguida por Medicina (1940). Actualmente, solo cuatro disciplinas médicas en el país comparten nuestro privilegio.

Asimismo, es de hacer notar que entre 1905 y 1929 Archivos se denominaba Archivos LatinoAmericanos de Pediatría, publicación conjunta con la Sociedad Uruguaya de Pediatría más la colaboración de autores brasileros y chilenos.

Desde la Subcomisión de Ética Clínica de la SAP tuvimos la intención de proponer y colaborar en un nuevo recurso que se sume a los actuales, todos con el mismo objetivo, facilitar el acceso de los lectores a información útil.

Se trata de lo que se decidió denominar "Colecciones", recurso equivalente al utilizado por prestigiosas publicaciones (Collections en Journal of Pediatrics, Pediatrics, JAMA Pediatrics, Arch. Dis.
Childhood, "Specialties \& Topics" en New England Journal of Medicine, etc.).

Para ello hemos confeccionado una "Colección de Bioética" con los artículos del tema publicados en Archivos.

Para ello se revisó PubMed ${ }^{\circledR}$ y el sitio web de la SAP. La estrategia de búsqueda usada en PubMed ${ }^{\circledR}$ fue: "Archivos Argentinos de Pediatria"[Journal] que devolvió 1397 ítems. Se revisaron 833 títulos desde el número de agosto de 2014 inclusive hasta el de febrero de 2008. Al momento de la revisión (octubre de 2014) el último artículo indexado con continuidad en PubMed era de febrero de 2008, saltando después a 1972, por lo que desde esa cita hacia atrás en el tiempo se siguió revisando la web SAP/Archivos (http:// sap.org.ar/publi-arch-numerosanteriores.php?menu=item3). Esta revisión agregó entonces desde el año 1998 hasta el 2007 completos. No había en ese momento registros anteriores a 1998 en el sitio web.

El criterio de selección de los artículos fue amplio, incluyendo filosofía, antropología, investigación e incluso algunos de política sanitaria. Decidimos esta amplitud porque nos pareció que era más fácil posteriormente excluir uno de esta
Correspondencia:

Dr. Jorge Selandari: jselandari@intramed. net

Recibido: 6-7-2015 Aceptado: 8-7-2015
Subcomisión de Ética Clínica - Sociedad Argentina de Pediatría: Dres. Jorge Selandari, Horacio Repetto, Gonzalo Pérez Marc, María C. Orsi, Diana Cohen Agrest, Alejandro Barceló, Lidia Albano, Patricio Cudeiro, María M. Cuneo, Fernando Ledesma, María del C. Martínez Perea y Miguel Del Valle. 
lista, que incluir uno que quedaba en la maraña de más de 800 artículos revisados para esta tarea.

Por otro lado, estamos lejos de garantizar que no se omitió en la revisión algún artículo que debía estar. Adicionalmente, otras fuentes de error/omisión halladas son: a) en PubMed hay citas "citation in process" que no se revisaron; b) en Web de SAP / Archivos hay en los años más anteriores, links a "Cartas al Editor" sin los títulos del contenido, que tampoco se revisaron. Por estos motivos invitamos a los lectores que de detectar alguna omisión se pongan en contacto con los editores o con la subcomisión de ética clínica para subsanarlos.

En PubMed se seleccionaron 67 artículos y en la web otros 53, totalizando 120 artículos.

Dada la cantidad de artículos seleccionados, como así también la gran amplitud del campo de la bioética, y con el objetivo de facilitar el acceso, hemos seleccionados algunos artículos en forma de "introducción" a la Colección.

Para ello subdividimos arbitrariamente tres escenarios:

- Microética, vinculado con la relación y toma de decisiones directa sobre/con el paciente ${ }^{1-18}$

- Mesoética, vinculada con las relaciones entre pares, la Pediatría como profesión y otros temas a nivel institucional ${ }^{19-32} \mathrm{y}$;

- Macroética, fundamentalmente relacionado con la vigilancia y defensa de los derechos de los niños, pero también con las concepciones antropológicas, sociales, filosóficas, etc. de la niñez y la adolescencia. ${ }^{33-51}$

Las temáticas incluyen tanto el escenario de internación como el paciente ambulatorio, el de

Figura 1. Fundamentos teóricos y metodológicos de la bioética como Competencia Clínica

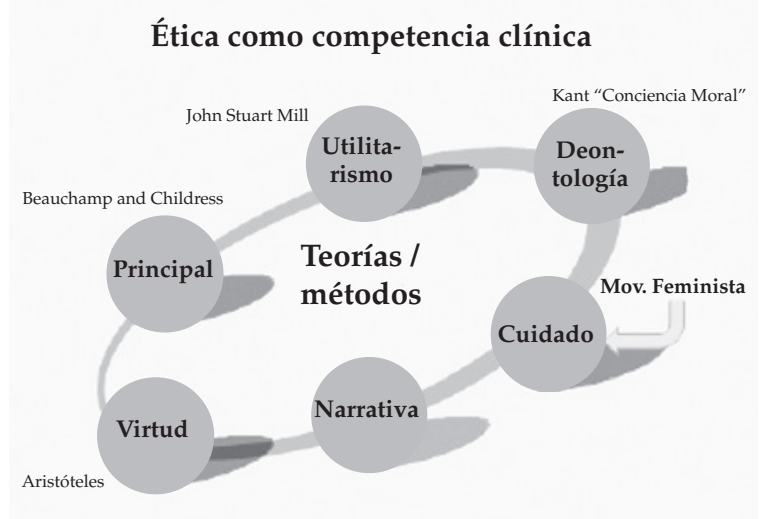

alta complejidad como el de atención primaria, el principio de la vida como la muerte en la edad pediátrica, la actividad clínica aislada como la investigación clínica.

Creemos que es útil considerar al ejercicio de la micro-bioética, en la relación asistencial entre el equipo de salud, el niño y su familia, como una competencia clínica más, que con fundamentos teóricos y metodológicos (ver algunos en la Figura 1), se debe cultivar para adquirir las habilidades y destrezas necesarias (ver algunas en el Cuadro 1) para resolver efectivamente este aspecto de los problemas clínicos. Pero también es importante para nosotros, los pediatras, incrementar continuamente nuestra cultura e idoneidad en los temas meso y macroéticos, de manera que progresivamente podamos responder mejor a nuestro deseo y a las expectativas de la sociedad: ser efectivos promotores, recuperadores, cuidadores y defensores de la salud, el bienestar y los derechos de los niños y adolescentes.

En fin, deseamos que esta sea una contribución positiva y un recurso útil a los lectores de Archivos.

\section{REFERENCIAS}

1. Puga T. Bioética en Pediatría. Arch Argent Pediatr 1999;97(1): 26-35.

2. Burbinski B, Naser M. Reflexiones acerca de la relación médico-paciente. Arch Argent Pediatr 1999;97(1):43-6.

3. Braga ML, Tarantino MG. La comunicación en pediatría: niñas, niños y adolescentes, sujetos de derecho. Arch Argent Pediatr 2011;109(1):36-41.

4. Comité Nacional de Terapia Intensiva Pediátrica. Consenso sobre recomendaciones acerca de la limitación del soporte vital en terapia intensiva. Arch Argent Pediatr 1999;97(6): 411-5.

5. Comité de Ética Hospitalaria. Hospital de Pediatría "Prof. Dr. J. P. Garrahan". Recomendaciones para la toma de decisiones terapéuticas en pacientes gravemente enfermos. Arch Argent Pediatr 1998;96(6):399-403.

Cuadro 1. Competencias en ética médica
$>$ Conocimientos
$\checkmark$ Teorías
$\checkmark$ Principios
$\checkmark$ Técnicas
$>$ Tolerancia y respeto
$\checkmark$ Habilidades sociales y emocionales
$>$ Comunicación efectiva
$>$ Deliberación moral
$>$ Mediación
$>$ etc. 
6. Silva TP. ¿Eutanasia o limitación del esfuerzo terapéutico? Arch Argent Pediatr 2012;110(3):203-4.

7. Cardigni G. Relación médico-familia ante la muerte de un niño en terapia intensiva pediátrica: no alcanza con buenas intenciones. Arch Argent Pediatr 2008;106(6):487-9.

8. Scrigni AV. Reflexiones sobre la inutilidad del tratamiento médico. Arch Argent Pediatr 2007;105(5):385-6.

9. Ghiggi M, Chede C, Saporiti A. De lo que no se habla en la UCIP. Arch Argent Pediatr 2001;99(4):337-9.

10. Ceriani Cernadas JM. La morbilidad reemplaza a la mortalidad: un dilema ético en el cuidado de los prematuros en los límites de la viabilidad. Arch Argent Pediatr 2012;110(2): 98-9.

11. Kipper D. ¿Hasta dónde los padres tienen derecho a decidir por sus hijos? Arch Argent Pediatr 1999;97(1):18-25.

12. Giglio N,MalozowskiS. Prescripciones fuera de prospecto. Arch Argent Pediatr 2004;102(2):121-4.

13. Comité Nacional de Endocrinología de la Sociedad Argentina de Pediatría. Consideraciones para el manejo inicial del recién nacido con ambigüedad genital. Arch Argent Pediatr 2004;102(2):148-51.

14. Sebastiani M,Ceriani Cernadas JM. Aspectos bioéticos en el cuidado de los recién nacidos extremadamente prematuros. Arch Argent Pediatr 2008;106(3):242-8.

15. Subcomisión de Ética Clínica. Embarazos en niñas y adolescentes. Arch Argent Pediatr 2010;108(6):562-5.

16. Escobar ME. Anticoncepción de emergencia. Arch Argent Pediatr 2003;101(6):430-1.

17. Schon A. El cuerpo en la intervención clínica. Una mirada desde los derechos del niño. Arch Argent Pediatr 2008;106(4):359-60.

18. Carrio S, De Cunto CL, Cacchiarelli N, Ceriani Cernadas $\mathrm{C}$, et al. Medicina narrativa en Pediatría: relato de una experiencia. Arch Argent Pediatr 2008;106(2):138-42.

19. Boggiano E. El pediatra: médico de cabecera de niños y adolescentes. Arch Argent Pediatr 2000;98(3):153-4.

20. de Asúa M. La pediatría como disciplina cultural y social. Arch Argent Pediatr 2012;110(3):231-6.

21. Braun R. Una visión holística de la pediatría. Arch Argent Pediatr 2011;109(6):504-9.

22. Lejarraga H. La interacción entre los médicos: consulta, referencia, contrarreferencia. Arch Argent Pediatr 2001;99(5): 385-6.

23. Sittig DF, Singh H, Longhurst CA. Derechos y responsabilidades de los usuarios de una historia clínica electrónica. Arch Argent Pediatr 2013;111(6):469-71.

24. Ceriani Cernadas JM. El cambio de cultura en el abordaje del error en medicina. ¿Cuánto hemos progresado? Arch Argent Pediatr 2013;111(2):90-1.

25. Althabe M, Ledesma F, Cernadas C, Flores C. Actitudes de los profesionales de salud frente a las decisiones de limitación o retiro de tratamiento en pediatría. Arch Argent Pediatr 2003;101(2):85-92.

26. Ferrero F, Moreno L. La ética en la enseñanza de la pediatría. Arch Argent Pediatr 2008;106(3):196-7.

27. Lejarraga $\mathrm{H}$. El desarrollo del sentido ético en el niño y la enseñanza de la pediatría. Arch Argent Pediatr 2008;106(5): 422-8.

28. Ceriani Cernadas JM. El camino hacia una medicina más humana. Arch Argent Pediatr 2013;111(4):274-5.
29. Agrest A. La actividad asistencial y los conocimientos. Arch Argent Pediatr 2010;108(5):386.

30. Jaim Etcheverry G. El debate entre la ciencia y el arte de la medicina. Arch Argent Pediatr 2011;109(4):290-1.

31. Pérez Marc G. Sujeto y dolor: introducción a una filosofía de la medicina. Arch Argent Pediatr 2010;108(5):434-7.

32. Ossorio MF, Ferrero F. Plagio en las publicaciones científicas. Arch Argent Pediatr 2010;108(2):103-4.

33. Ceriani Cernadas JM. La tecnología médica como objeto de la ética. Arch Argent Pediatr 2014;112(4):298-9.

34. Ceriani Cernadas JM. Mejor medicina o más medicina, ¿hacia dónde vamos? Arch Argent Pediatr 2013;111(5):370-

35. Rovere M. La pediatría y la construcción social dela infancia. Análisis y perspectivas. Arch Argent Pediatr 2013;111(3): 224-31.

36. Rossato N. Legislación anti-tabáquica y prematurez. Arch Argent Pediatr 2013;111(3):186-7.

37. Equipo de salud Hospital Tornú. Análisis del fallo de la Corte Suprema de la República Argentina despenalizando el aborto. Arch Argent Pediatr 2013;111(2):181.

38. Rossato N. La muerte súbita de un lactante. No se buscan culpables sino quienes se hagan cargo de la prevención. Arch Argent Pediatr 2013;111(1):3-4.

39. Cutri A, Hammermüller E, Zubieta A, Müller Opet B, Miguelez L. Trabajo infantil: una problemática social que nos compromete. Arch Argent Pediatr 2012;110(4):350-8.

40. Rossato N. Pesquisa neonatal obligatoria. Reflexiones. Arch Argent Pediatr 2009;107(3):193-4.

41. Ramonet M. Repensar y acordar los Objetivos de Salud Materno-Infantil en la reforma de la Atención Primaria de la Salud. Una propuesta desde la Pediatría. Arch Argent Pediatr 2008;106(6):486.

42. Cardoso P, Calabró P. Investigación clínica farmacológica en pediatría: ¿Es ético y legal experimentar en niños? (Parte 1) Arch Arg Pediatr 2005;103(1):46-54.

43. Cardoso P, Calabró P. Investigación clínica farmacológica en pediatría: Consentimiento informado y asentimiento. ¿Quélugar tiene la voluntad del paciente pediátrico? (Parte 2) Arch Argent Pediatr 2005;103(2):135-146.

44. Longo D ¿Salud, educación y equidad en pediatría? Arch Argent Pediatr 2005;103(2):97-8.

45. Lejarraga H. Los derechos del niño y el rol del pediatra Arch Argent Pediatr 2005;103(4):344-7.

46. Beltramino D, Ramonet M. Los derechos de niños, niñas y adolescentes en la Argentina. Arch Argent Pediatr 2004;102(5):321-2.

47. Puga T. Los derechos del niño T. Arch Argent Pediatr 2001;99(2):150-61.

48. Sonis A. Equidad y salud. Arch Argent Pediatr 2001;99(3): 253-6.

49. Riterman F. El derecho a la salud, a la vida, a la identidad. Arch Argent Pediatr 2000;98(3):199-200.

50. Altamirano F, Arcusin J, Pasamonic J, Pignata N, et al. El derecho a la salud: asistencia y juridicidad en la atención de niños, niñas y adolescentes en situación de calle. Arch Argent Pediatr 2004;102(3):220-9.

51. Wahren C, De Cunto C. La publicidad en las revistas médicas, esa delgada línea roja. Arch Argent Pediatr 2004;102(6):422-4. 